\title{
Fourier series of higher-order Daehee and Changhee functions and their applications
}

\section{Dongkyu Lim* (10}

\section{"Correspondence:}

dgrim84@gmail.com

School of Mathematical Sciences,

Nankai University, Tianjin City,

300071, China

\section{Abstract}

In the paper, the author considers the Fourier series related to higher-order Daehee and Changhee functions and establishes some new identities for higher-order Daehee and Changhee functions.

MSC: $11 \mathrm{~B} 68 ; 42 \mathrm{~A} 16$

Keywords: Fourier series; Daehee polynomials; Changhee polynomials; Bernoulli functions; Daehee functions; Changhee functions

\section{Introduction and main results}

It is common knowledge that the Bernoulli polynomials $B_{n}(x)$ and the Euler polynomials $E_{n}(x)$ for $n \geq 0$ can be generated by

$$
\frac{t}{e^{t}-1} e^{x t}=\sum_{n=0}^{\infty} B_{n}(x) \frac{t^{n}}{n !}
$$

and

$$
\frac{2}{e^{t}+1} e^{x t}=\sum_{n=0}^{\infty} E_{n}(x) \frac{t^{n}}{n !},
$$

respectively (see [1-23]).

With the viewpoint of deformed Bernoulli polynomials, the Daehee polynomials $D_{n}(x)$ for $n \geq 0$ are defined by the generating function to be

$$
\frac{\log (1+t)}{t}(1+t)^{x}=\sum_{n=0}^{\infty} D_{n}(x) \frac{t^{n}}{n !}
$$

It is easy to see that the generating function of the Daehee polynomials $D_{n}(x)$ can be reformed as

$$
\frac{\log (1+t)}{t}(1+t)^{x}=\frac{\log (1+t)}{e^{\log (1+t)}-1} e^{x \log (1+t)}
$$

(c) The Author(s) 2017. This article is distributed under the terms of the Creative Commons Attribution 4.0 International License (http://creativecommons.org/licenses/by/4.0/), which permits unrestricted use, distribution, and reproduction in any medium, provided you give appropriate credit to the original author(s) and the source, provide a link to the Creative Commons license, and indicate if changes were made. 
From (1), we note that

$$
\begin{aligned}
\frac{\log (1+t)}{e^{\log (1+t)}-1} e^{x \log (1+t)} & =\sum_{n=0}^{\infty} B_{n}(x) \frac{1}{n !}(\log (1+t))^{n} \\
& =\sum_{n=0}^{\infty} B_{n}(x) \sum_{m=n}^{\infty} S_{1}(m, n) \frac{t^{m}}{m !} \\
& =\sum_{m=0}^{\infty}\left(\sum_{n=0}^{m} B_{n}(x) S_{1}(m, n)\right) \frac{t^{m}}{m !},
\end{aligned}
$$

where $S_{1}(m, n)$ stands for the Stirling number of the first kind which is defined as

$$
(x)_{0}=1, \quad(x)_{n}=x(x-1) \cdots(x-n+1)=\sum_{l=0}^{n} S_{1}(n, l) x^{l} \quad(n \geq 1) .
$$

Combining (1) with (2) yields the following relation:

$$
D_{m}(x)=\sum_{n=0}^{m} B_{n}(x) S_{1}(m, n) \quad(m \geq 0)
$$

By replacing $t$ by $e^{t}-1$ in (1), we can derive

$$
\begin{aligned}
\sum_{n=0}^{\infty} B_{n}(x) \frac{t^{n}}{n !} & =\frac{t}{e^{t}-1} e^{x t}=\sum_{m=0}^{\infty} D_{m}(x) \frac{1}{m !}\left(e^{t}-1\right)^{m} \\
& =\sum_{m=0}^{\infty} D_{m}(x) \sum_{n=m}^{\infty} S_{2}(n, m) \frac{t^{n}}{n !} \\
& =\sum_{n=0}^{\infty}\left(\sum_{m=0}^{n} D_{n}(x) S_{2}(n, m)\right) \frac{t^{n}}{n !}
\end{aligned}
$$

where $S_{2}(n, m)$ is the Stirling number of the second kind which is given by $x^{n}=$ $\sum_{l=0}^{\infty} S_{2}(n, l)(x)_{l}(n \geq 0)$.

Comparing the coefficients on the both sides of (3), we obtain

$$
B_{n}(x)=\sum_{m=0}^{n} D_{m}(x) S_{2}(n, m) \quad(n \geq 0)
$$

Also, with the viewpoint of deformed Euler polynomials, the Changhee polynomials $C h_{n}(x)$ for $n \geq 0$ are defined by the generating function to be

$$
\frac{2}{2+t}(1+t)^{x}=\sum_{n=0}^{\infty} C h_{n}(x) \frac{t^{n}}{n !}
$$


Definition (4) can be written as

$$
\begin{aligned}
\frac{2}{e^{\log (1+t)}+1} e^{x \log (1+t)} & =\sum_{n=0}^{\infty} E_{n}(x) \frac{1}{n !}(\log (1+t))^{n} \\
& =\sum_{n=0}^{\infty} E_{n}(x) \sum_{m=n}^{\infty} S_{1}(m, n) \frac{t^{m}}{m !} \\
& =\sum_{m=0}^{\infty}\left(\sum_{n=0}^{m} E_{n}(x) S_{1}(m, n)\right) \frac{t^{m}}{m !} .
\end{aligned}
$$

Combination of this identity with (4) results in the following relation:

$$
C h_{m}(x)=\sum_{n=0}^{m} E_{n}(x) S_{1}(m, n) \quad(m \geq 0) .
$$

Now replacing $t$ by $e^{t}-1$ in (4), we have

$$
\begin{aligned}
\sum_{n=0}^{\infty} E_{n}(x) \frac{t^{n}}{n !} & =\frac{2}{e^{t}+1} e^{x t}=\sum_{m=0}^{\infty} C h_{m}(x) \frac{1}{m !}\left(e^{t}-1\right)^{m} \\
& =\sum_{m=0}^{\infty} C h_{m}(x) \sum_{n=m}^{\infty} S_{2}(n, m) \frac{t^{n}}{n !} \\
& =\sum_{n=0}^{\infty}\left(\sum_{m=0}^{n} C_{n}(x) S_{2}(n, m)\right) \frac{t^{n}}{n !}
\end{aligned}
$$

Equating coefficients on the very ends of the above identity leads to

$$
E_{n}(x)=\sum_{m=0}^{n} C h_{m}(x) S_{2}(n, m) \quad(n \geq 0) .
$$

In recent decades, many mathematicians have investigated some interesting extensions or modifications of the Daehee and Changhee polynomials along with related combinatorial identities and their applications (see $[4,9,10,14,16,17,19,23])$. Especially, Kim and his coauthors have studied the Fourier series related to various types of Bernoulli functions in $[7,11-13,15]$. The purpose of this paper is to study the Fourier series related to higherorder Daehee and Changhee functions and establish some new identities for higher-order Daehee and Changhee functions.

For any real number $x$, we define

$$
\langle x\rangle=x-[x] \in(0,1)
$$

where $[x]$ is the integer part of $x$. Then $D_{n}(\langle x\rangle)$ are functions defined on $(-\infty, \infty)$ and periodic with period 1 , which are called Daehee functions.

For $r \in \mathbb{N}$ and $n \geq 0$, we note that the higher-order Daehee polynomials $D_{n}^{(r)}(x)$ and the higher-order Changhee polynomials $C h_{n}^{(r)}(x)$ may also be represented by the following 
generating function:

$$
\left(\frac{\log (1+t)}{t}\right)^{r}(1+t)^{x}=\sum_{n=0}^{\infty} D_{n}^{(r)}(x) \frac{t^{n}}{n !}
$$

and

$$
\left(\frac{2}{2+t}\right)^{r}(1+t)^{x}=\sum_{n=0}^{\infty} C h_{n}^{(r)}(x) \frac{t^{n}}{n !}
$$

respectively (see $[4,10,14])$. When $x=0, D_{n}^{(r)}=D_{n}^{(r)}(0)$ are called the higher-order Daehee numbers and $C h_{n}^{(r)}=C h_{n}^{(r)}(0)$ are called the higher-order Changhee numbers. And it is easy to see that

$$
D_{n}^{(1)}(x)=D_{n}(x), \quad C h_{n}^{(1)}(x)=C h_{n}(x) .
$$

Then $D_{n}^{(r)}(\langle x\rangle)$ and $C h_{n}^{(r)}(\langle x\rangle)$ are functions defined on $(-\infty, \infty)$ and periodic of period 1, which are called Daehee functions of order $r$ and Changhee functions of order $r$, respectively.

Recall from $[15,24]$ that the Bernoulli function may be represented by

$$
B_{m}(\langle x\rangle)=-m ! \sum_{\substack{n=-\infty \\ n \neq 0}}^{\infty} \frac{e^{2 \pi i n x}}{(2 \pi i n)^{m}} \quad(m \geq 2)
$$

and

$$
-m ! \sum_{\substack{n=-\infty \\ n \neq 0}}^{\infty} \frac{e^{2 \pi i n x}}{(2 \pi i n)^{m}}= \begin{cases}B_{1}(\langle x\rangle) & \text { for } x \notin \mathbb{Z}, \\ 0 & \text { for } x \in \mathbb{Z} .\end{cases}
$$

The Fourier series expansion of the Bernoulli functions is useful in computing the special values of the Dirichlet $L$-functions. For details, one is referred to [24].

Our main results in this paper can be stated as the following theorems.

Theorem 1 Let $m \geq 2, r \geq 1$. Assume that $D_{m-1}^{(r)}=0$.

(a) $D_{m}^{(r)}(\langle x\rangle)$ has the Fourier series expansion

$$
D_{m}^{(r)}(\langle x\rangle)=D_{m}^{(r)}-\sum_{\substack{n=-\infty \\ n \neq 0}}^{\infty}\left(\sum_{k=1}^{m} \frac{(m)_{k}}{(2 \pi i n)^{k}} D_{m-k}^{(r)}\right) e^{2 \pi i n x}
$$

for $x \in(-\infty, \infty)$. Here the convergence is uniform.

(b) $D_{m}^{(r)}(\langle x\rangle)=\sum_{\substack{k=0 \\ k \neq 1}}^{m}\left(\begin{array}{c}m \\ k\end{array}\right) D_{m-1}^{(r)} B_{k}(\langle x\rangle)$, for all $x \in(-\infty, \infty)$, where $B_{k}(\langle x\rangle)$ is the Bernoulli function. 
Theorem 2 Let $m \geq 2, r \geq 1$. Assume that $D_{m-1}^{(r)} \neq 0$.

(a)

$$
D_{m}^{(r)}-\sum_{\substack{n=-\infty \\ n \neq 0}}^{\infty}\left(\sum_{k=1}^{m} \frac{(m)_{k}}{(2 \pi i n)^{k}} D_{m-k}^{(r)}\right) e^{2 \pi i n x}= \begin{cases}D_{m}^{(r)}(\langle x\rangle) & \text { for } x \notin \mathbb{Z}, \\ D_{m}^{(r)}+\frac{m}{2} D_{m-1}^{(r)} & \text { for } x \in \mathbb{Z} .\end{cases}
$$

Here the convergence is pointwise.

(b)

$$
\sum_{k=0}^{m}\left(\begin{array}{c}
m \\
k
\end{array}\right) D_{m-k}^{(r)} B_{k}(\langle x\rangle)=D_{m}^{(r)}(x) \quad \text { for } x \notin \mathbb{Z}
$$

and

$$
\sum_{\substack{k=0 \\
k \neq 1}}^{m}\left(\begin{array}{c}
m \\
k
\end{array}\right) D_{m-k}^{(r)} B_{k}(\langle x\rangle)=D_{m}^{(r)}+\frac{m}{2} D_{m-1}^{(r)} \quad \text { for } x \in \mathbb{Z},
$$

where $B_{k}(\langle x\rangle)$ is the Bernoulli function.

Theorem 3 Let $m \geq 2, r \geq 1$. Assume that $C h_{m}^{(r)}=C h_{m}^{(r-1)}$.

(a) $C h_{m}^{(r)}(\langle x\rangle)$ has the Fourier series expansion

$$
\begin{aligned}
C h_{m}^{(r)}(\langle x\rangle)= & \frac{2}{m+1}\left(C h_{m+1}^{(r-1)}-C h_{m+1}^{(r)}\right) \\
& +\sum_{\substack{n=-\infty \\
n \neq 0}}^{\infty}\left(\sum_{k=1}^{m} \frac{2(m)_{k-1}}{(2 \pi i n)^{k}}\left(C h_{m-k+1}^{(r)}-C h_{m-k+1}^{(r-1)}\right)\right) e^{2 \pi i n x}
\end{aligned}
$$

for $x \in(-\infty, \infty)$. Here the convergence is uniform.

(b)

$$
\begin{aligned}
C h_{m}^{(r)}(\langle x\rangle)= & \frac{2}{m+1}\left(C h_{m+1}^{(r)}-C h_{m+1}^{(r)}\right) \\
& +\sum_{k=1}^{m} \frac{2(m)_{k-1}}{k !}\left(C h_{m-k+1}^{(r-1)}-C h_{m+1}^{(r)}\right) B_{k}(\langle x\rangle) \quad \text { for } x \notin \mathbb{Z}
\end{aligned}
$$

and

$$
\begin{aligned}
C h_{m}^{(r)}(\langle x\rangle)= & \frac{2}{m+1}\left(C h_{m+1}^{(r-1)}-C h_{m-k+1}^{(r)}\right) \\
& +\sum_{k=2}^{m} \frac{2(m)_{k-1}}{k !}\left(C h_{m-k+1}^{(r-1)}-C h_{m+1}^{(r)}\right) B_{k}(\langle x\rangle) \quad \text { for } x \in \mathbb{Z},
\end{aligned}
$$

where $B_{k}(\langle x\rangle)$ is the Bernoulli function. 
Theorem 4 Let $m \geq 1, r \geq 1$. Assume that $C h_{m}^{(r)} \neq C h_{m}^{(r-1)}$.

(a)

$$
\begin{aligned}
& \frac{2}{m+1}\left(C h_{m+1}^{(r-1)}-C h_{m+1}^{(r)}\right)+\sum_{\substack{n=-\infty \\
n \neq 0}}^{\infty}\left(\sum_{k=1}^{n} \frac{(m)_{k-1}}{(2 \pi i n)^{k}}\left(C h_{m-k+1}^{(r)}-C h_{m-k+1}^{(r-1)}\right)\right) e^{2 \pi i n x} \\
& \quad= \begin{cases}C h_{m}^{(r)}(\langle x\rangle) & \text { for } x \notin \mathbb{Z}, \\
C h_{m}^{(r-1)} & \text { for } x \in \mathbb{Z} .\end{cases}
\end{aligned}
$$

Here the convergence is pointwise.

(b)

$$
\begin{aligned}
& \frac{2}{m+1}\left(C h_{m+1}^{(r-1)}-C h_{m+1}^{(r)}\right)+\sum_{k=1}^{m} \frac{2(m)_{k-1}}{k !}\left(C h_{m-k+1}^{(r-1)}-C h_{m-k+1}^{(r)}\right) B_{k}(\langle x\rangle) \\
& \quad=C h_{m}^{(r)}(\langle x\rangle) \quad \text { for } x \notin \mathbb{Z}
\end{aligned}
$$

and

$$
\begin{aligned}
& \frac{2}{m+1}\left(C h_{m+1}^{(r-1)}-C h_{m+1}^{(r)}\right)+\sum_{k=2}^{m} \frac{2(m)_{k-1}}{k !}\left(C h_{m-k+1}^{(r-1)}-C h_{m-k+1}^{(r)}\right) B_{k}(\langle x\rangle) \\
& =C h_{m}^{(r-1)}(\langle x\rangle) \quad \text { for } x \in \mathbb{Z}
\end{aligned}
$$

where $B_{k}(\langle x\rangle)$ is the Bernoulli function.

\section{Proofs of Theorems 1-4}

We are now in a position to prove our four theorems.

By analyzing definition (5), we have

$$
D_{m}^{(r)}(x+1)=D_{m}^{(r)}(x)+m D_{m-1}^{(r)}(x) \quad(m \geq 0) .
$$

Furthermore, we observe that

$$
\begin{aligned}
\sum_{m=0}^{\infty} D_{m}^{(r)}(x) \frac{t^{m}}{m !} & =\left(\frac{\log (1+t)}{t}\right)^{r}(1+t)^{x+1} \\
& =\left(\frac{\log (1+t)}{t}\right)^{r}(1+t)^{x}+\left(\frac{\log (1+t)}{t}\right)^{r}(1+t)^{x} t \\
& =\sum_{m=0}^{\infty} D_{m}^{(r)}(x) \frac{t^{m}}{m !}+\sum_{m=0}^{\infty} D_{m}^{(r)}(x) \frac{t^{m+1}}{m !} \\
& =\sum_{m=0}^{\infty}\left(D_{m}^{(r)}(x)+m D_{m-1}^{(r)}(x)\right) \frac{t^{m}}{m !} .
\end{aligned}
$$

Letting $x=0$ in the above equation leads to

$$
D_{m}^{(r)}(1)=D_{m}^{(r)}+m D_{m-1}^{(r)} \quad(m \geq 0) .
$$


Now, we assume that $m, r \geq 1 . D_{m}^{(r)}(\langle x\rangle)$ is piecewise $C^{\infty}$. Further, in view of $(2), D_{m}^{(r)}(\langle x\rangle)$ is continuous for those $(r, m)$ with $D_{m-1}^{(r)}=0$, and is discontinuous with jump discontinuities at integers for those $(r, m)$ with $D_{m-1}^{(r)} \neq 0$. The Fourier series of $D_{m}^{(r)}(\langle x\rangle)$ may be represented by

$$
\sum_{n=-\infty}^{\infty} C_{n}^{(r, m)} e^{2 \pi i n x} \quad(i=\sqrt{-1})
$$

where

$$
\begin{aligned}
C_{n}^{(r, m)} & =\int_{0}^{1} D_{m}^{(r)}(\langle x\rangle) e^{-2 \pi i n x} d x=\int_{0}^{1} D_{m}^{(r)}(x) e^{-2 \pi i n x} d x \\
& =\left[\frac{1}{m+1} D_{m+1}^{(r)}(x) e^{-2 \pi i n x}\right]_{0}^{1}+\frac{2 \pi i n}{m+1} \int_{0}^{1} D_{m+1}^{(r)}(x) e^{-2 \pi i n x} d x \\
& =\frac{1}{m+1}\left(D_{m+1}^{(r)}(1)-D_{m+1}^{(r)}\right)+\frac{2 \pi i n}{m+1} C_{n}^{(r, m+1)} \\
& =D_{m}^{(r)}+\frac{2 \pi i n}{m+1} C_{n}^{(r, m+1)} .
\end{aligned}
$$

Replacing $m$ by $m-1$ in (9), we arrive at the following result:

$$
C_{n}^{(r, m-1)}=D_{m-1}^{(r)}+\frac{2 \pi i n}{m} C_{n}^{(r, m)}
$$

Case 1 Let $n \neq 0$. Then we acquire that

$$
\begin{aligned}
C_{n}^{(r, m)}= & \frac{m}{2 \pi i n} C_{n}^{(r, m-1)}-\frac{m}{2 \pi i n} D_{m-1}^{(r)} \\
= & \frac{m}{2 \pi i n}\left(\frac{m-1}{2 \pi i n} C_{n}^{(r, m-2)}-\frac{m-1}{2 \pi i n} D_{m-2}^{(r)}\right)-\frac{m}{2 \pi i n} D_{m-1}^{(r)} \\
= & \frac{m(m-1)}{(2 \pi i n)^{2}} C_{n}^{(r, m-2)}-\frac{m(m-1)}{(2 \pi i n)^{2}} D_{m-2}^{(r)}-\frac{m}{2 \pi i n} D_{m-1}^{(r)} \\
= & \frac{m(m-1)}{(2 \pi i n)^{2}}\left(\frac{m-2}{2 \pi i n} C_{n}^{(r, m-3)}-\frac{m-2}{2 \pi i n} D_{m-3}^{(r)}\right) \\
& -\frac{m(m-1)}{(2 \pi i n)^{2}} D_{m-2}^{(r)}-\frac{m}{2 \pi i n} D_{m-1}^{(r)} \\
= & \frac{m(m-1)(m-2)}{(2 \pi i n)^{2}} C_{n}^{(r, m-3)}-\frac{m(m-1)(m-2)}{(2 \pi i n)^{3}} D_{m-3}^{(r)} \\
& -\frac{m(m-1)}{(2 \pi i n)^{2}} D_{m-2}^{(r)}-\frac{m}{2 \pi i n} D_{m-1}^{(r)} \\
= & \cdots \\
= & \frac{m(m-1)(m-2) \cdots 2}{(2 \pi i n)^{m-1}} C_{n}^{(r, 1)}-\sum_{k=1}^{m-1} \frac{(m)_{k}}{(2 \pi i n)^{k}} D_{m-k}^{(r)} \cdot
\end{aligned}
$$


Moreover, we observe that

$$
\begin{aligned}
C_{n}^{(r, 1)} & =\int_{0}^{1} D_{1}^{(r)}(x) e^{-2 \pi i n x} d x=\int_{0}^{1}\left(x+D_{1}^{(r)}\right) e^{-2 \pi i n x} d x \\
& =\int_{0}^{1} x e^{-2 \pi i n x} d x+D_{1}^{(r)} \int_{0}^{1} e^{-2 \pi i n x} d x \\
& =-\frac{1}{2 \pi i n}\left[x e^{-2 \pi i n x}\right]_{0}^{1}+\frac{1}{2 \pi i n} \int_{0}^{1} e^{-2 \pi i n x} d x=-\frac{1}{2 \pi i n} .
\end{aligned}
$$

Combining (11) with (10), we immediately derive the following equation:

$$
C_{n}^{(r, m)}=\frac{m !}{(2 \pi i n)^{m}}-\sum_{k=1}^{m-1} \frac{(m)_{k}}{(2 \pi i n)^{k}} D_{m-k}^{(r)}=-\sum_{k=1}^{m} \frac{(m)_{k}}{(2 \pi i n)^{k}} D_{m-k}^{(r)} .
$$

Case 2 Let $n=0$. Then we have

$$
\begin{aligned}
C_{0}^{(r, m)} & =\int_{0}^{1} D_{m}^{(r)}(\langle x\rangle) d x=\int_{0}^{1} D_{m}^{(r)}(x) d x \\
& =\frac{1}{m+1}\left[D_{m+1}^{(r)}(x)\right]_{0}^{1} \\
& =\frac{1}{m+1}\left(D_{m+1}^{(r)}(1)-D_{m+1}^{(r)}\right)=D_{m}^{(r)} .
\end{aligned}
$$

While that in (8) converges pointwise, the series in (7) converges uniformly. We assume that $D_{m-1}^{(r)}=0$. Then we have $D_{m}^{(r)}(1)=D_{m}^{(r)}$ for $m \geq 2$. As $D_{m}^{(r)}(\langle x\rangle)$ is piecewise $C^{\infty}$ and continuous, the Fourier series of $D_{m}^{(r)}(\langle x\rangle)$ converges uniformly to $D_{m}^{(r)}(\langle x\rangle)$ and

$$
\begin{aligned}
& D_{m}^{(r)}(\langle x\rangle)=\sum_{n=-\infty}^{\infty} C_{n}^{(r, m)} e^{2 \pi i n x} \\
& =D_{m}^{(r)}-\sum_{\substack{n=-\infty \\
n \neq 0}}^{\infty}\left(\sum_{k=1}^{m} \frac{(m)_{k}}{(2 \pi i n)^{k}} D_{m-k}^{(r)}\right) e^{2 \pi i n x} \\
& =D_{m}^{(r)}+\sum_{k=1}^{m} \frac{(m)_{k}}{k !} D_{m-k}^{(r)}\left(k ! \sum_{\substack{n=-\infty \\
n \neq 0}}^{\infty} \frac{e^{2 \pi i n x}}{(2 \pi i n)^{k}}\right) \\
& =D_{m}^{(r)}+\sum_{k=2}^{m}\left(\begin{array}{c}
m \\
k
\end{array}\right) D_{m-k}^{(r)} B_{k}(\langle x\rangle)+\left(\begin{array}{c}
m \\
1
\end{array}\right) D_{m-1}^{(r)} \times \begin{cases}B_{1}(\langle x\rangle) & \text { for } x \notin \mathbb{Z}, \\
0 & \text { for } x \in \mathbb{Z}\end{cases} \\
& = \begin{cases}\sum_{k=0}^{m}\left(\begin{array}{c}
m \\
k
\end{array}\right) D_{m-1}^{(r)} B_{k}(\langle x\rangle) & \text { for } x \notin \mathbb{Z} \\
\sum_{\substack{k=0 \\
k \neq 1}}^{m}\left(\begin{array}{c}
m \\
k
\end{array}\right) D_{m-1}^{(r)} B_{k}(\langle x\rangle) & \text { for } x \in \mathbb{Z}\end{cases}
\end{aligned}
$$

Note that (12) holds whether $D_{m-1}^{(r)}=0$ or not. However, if $D_{m-1}^{(r-1)}=0$, then

$$
D_{m}^{(r)}(\langle x\rangle)=\sum_{\substack{k=0 \\
k \neq 1}}^{m}\left(\begin{array}{c}
m \\
k
\end{array}\right) D_{m-1}^{(r)} B_{k}(\langle x\rangle) \text { for all } x \in(-\infty, \infty)
$$

Therefore, we obtain the result in Theorem 1. 
Assume next that $D_{m-1}^{(r)} \neq 0$. Then we have $D_{m}^{(r)}(1) \neq D_{m}^{(r)}$ and hence $D_{m}^{(r)}(\langle x\rangle)$ is piecewise $C^{\infty}$ and discontinuous with jump discontinuities at integers. Thus the Fourier series of $D_{m}^{(r)}(\langle x\rangle)$ converges pointwise to $D_{m}^{(r)}(\langle x\rangle)$ for $x \notin \mathbb{Z}$, and converges to $\frac{1}{2}\left(D_{m}^{(r)}+D_{m}^{(r)}(1)\right)=$ $D_{m}^{(r)}+(m / 2) D_{m-1}^{(r)}$ for $x \in \mathbb{Z}$. Finally, we obtain the formulas in Theorem 2 .

From now on we focus on definition (6). Then we can find

$$
C h_{m}^{(r)}(x+1)+C h_{m}^{(r)}(x)=2 C h_{m}^{(r-1)}(x)
$$

In other words,

$$
\begin{aligned}
\sum_{m=0}^{\infty} C h_{m}^{(r)}(x+1) \frac{t^{m}}{m !} & =\left(\frac{2}{2+t}\right)^{r}(1+t)^{x+1} \\
& =2\left(\frac{2}{2+t}\right)^{r-1}(1+t)^{x}-\left(\frac{2}{2+t}\right)^{r}(1+t)^{x} \\
& =2 \sum_{m=0}^{\infty} C h_{m}^{(r-1)}(x) \frac{t^{m}}{m !}-\sum_{m=0}^{\infty} C h_{m}^{(r)}(x) \frac{t^{m}}{m !} \\
& =\sum_{m=0}^{\infty}\left[2 C h_{m}^{(r-1)}(x)-C h_{m}^{(r)}(x)\right] \frac{t^{m}}{m !} .
\end{aligned}
$$

Taking $x=0$ in (13) yields

$$
C h_{m}^{(r)}(1)+C h_{m}^{(r)}=2 C h_{m}^{(r-1)} \quad(m \geq 0)
$$

This equation means that

$$
C h_{m}^{(r)}=C h_{m}^{(r)}(1) \quad \Leftrightarrow \quad C h_{m}^{(r)}=C h_{m}^{(r-1)} .
$$

Assume that $m \geq 1$ and $r \geq 1 C h_{m}^{(r)}(\langle x\rangle)$ is piecewise $C^{\infty}$. In addition, $C h_{m}^{(r)}(\langle x\rangle)$ is continuous for those $(r, m)$ with $C h_{m}^{(r)}=C h_{m}^{(r-1)}$ and discontinuous with jump discontinuities at integers for those $(r, m)$ with $C h_{m}^{(r)} \neq C h_{m}^{(r-1)}$. The Fourier series of $C h_{m}^{(r)}(\langle x\rangle)$ is

$$
\sum_{n=-\infty}^{\infty} C_{n}^{(r, m)} e^{2 \pi i n x}
$$

Here

$$
\begin{aligned}
C_{n}^{(r, m)} & =\int_{0}^{1} C h_{m}^{(r)}(\langle x\rangle) e^{-2 \pi i n x} d x=\int_{0}^{1} C h_{m}^{(r)}(x) e^{-2 \pi i n x} d x \\
& =\frac{1}{m+1}\left[C h_{m+1}^{(r)}(x) e^{-2 \pi i n x}\right]_{0}^{1}+\frac{2 \pi i n}{m+1} \int_{0}^{1} C h_{m+1}^{(r)}(x) e^{-2 \pi i n x} d x \\
& =\frac{1}{m+1}\left(C h_{m+1}^{(r)}(1)-C h_{m+1}^{(r)}\right)+\frac{2 \pi i n}{m+1} C_{n}^{(r, m+1)} \\
& =\frac{2}{m+1}\left(C h_{m+1}^{(r-1)}-C h_{m+1}^{(r)}\right)+\frac{2 \pi i n}{m+1} C_{n}^{(r, m+1)} .
\end{aligned}
$$

By virtue of replacing $m$ by $m-1$ in (14), we can find

$$
\frac{2 \pi i n}{m} C_{n}^{(r, m)}=C_{n}^{(r, m-1)}+\frac{2}{m}\left(-C h_{m}^{(r-1)}+C h_{m}^{(r)}\right) .
$$


Case 1 Let $n \neq 0$. Then we acquire that

$$
\begin{aligned}
C_{n}^{(r, m)}= & \frac{m}{2 \pi i n} C_{n}^{(r, m-1)}+\frac{1}{\pi i n}\left(C h_{m}^{(r)}-C h_{m}^{(r-1)}\right) \\
= & \frac{m}{2 \pi i n}\left(\frac{m-1}{2 \pi i n} C_{n}^{(r, m-2)}-\frac{1}{\pi i n}\left(C h_{m-1}^{(r)}-C h_{m-1}^{(r-1)}\right)\right) \\
& +\frac{1}{\pi i n}\left(C h_{m}^{(r)}-C h_{m}^{(r-1)}\right) \\
= & \frac{m(m-1)}{(2 \pi i n)^{2}} C_{n}^{(r, m-2)}+\frac{m}{2(\pi i n)^{2}}\left(C h_{m-1}^{(r)}-C h_{m-1}^{(r-1)}\right) \\
& +\frac{1}{\pi i n}\left(C h_{m}^{(r)}-C h_{m}^{(r-1)}\right) \\
= & \frac{m(m-1)}{(2 \pi i n)^{2}}\left(\frac{m-2}{2 \pi i n} C_{n}^{(r, m-3)}-\frac{1}{\pi i n}\left(C h_{m-2}^{(r)}-C h_{m-2}^{(r-1)}\right)\right) \\
& +\frac{m}{2(\pi i n)^{2}}\left(C h_{m-1}^{(r)}-C h_{m-1}^{(r-1)}\right)+\frac{1}{\pi i n}\left(C h_{m}^{(r)}-C h_{m}^{(r-1)}\right) \\
= & \frac{m(m-1)(m-2)}{(2 \pi i n)^{3}} C_{n}^{(r, m-3)}+\frac{m(m-1)}{2^{2}(\pi i n)^{3}}\left(C h_{m-2}^{(r)}-C h_{m-2}^{(r-1)}\right) \\
& +\frac{m}{2(\pi i n)^{2}}\left(C h_{m-1}^{(r)}-C h_{m-1}^{(r-1)}\right)+\frac{1}{\pi i n}\left(C h_{m}^{(r)}-C h_{m}^{(r-1)}\right) \\
= & \cdots \\
= & \frac{m !}{(2 \pi i n)^{m-1}} C_{n}^{(r, 1)}+\sum_{k=1}^{m-1} \frac{2(m)_{k}}{(2 \pi i n)^{k}}\left(C h_{m-k+1}^{(r)}-C h_{m-k+1}^{(r-1)}\right) .
\end{aligned}
$$

In addition, we observe that

$$
\begin{aligned}
C_{n}^{(r, 1)} & =\int_{0}^{1} C h_{1}^{(r)}(x) e^{-2 \pi i n x} d x=\int_{0}^{1}\left(x+C h_{1}^{(r)}\right) e^{-2 \pi i n x} d x \\
& =\int_{0}^{1} x e^{-2 \pi i n x} d x+C h_{1}^{(r)} \int_{0}^{1} e^{-2 \pi i n x} d x \\
& =-\frac{1}{2 \pi i n}\left[x e^{-2 \pi i n x}\right]_{0}^{1}+\frac{1}{2 \pi i n} \int_{0}^{1} e^{-2 \pi i n x} d x \\
& =-\frac{1}{2 \pi i n} .
\end{aligned}
$$

Therefore, we can derive the following equation:

$$
\begin{aligned}
C_{n}^{(r, m)} & =\frac{-m !}{(2 \pi i n)^{m}}+\sum_{k=1}^{m-1} \frac{2(m)_{k-1}}{(2 \pi i n)^{k}}\left(C h_{m-k+1}^{(r)}-C h_{m-k+1}^{(r-1)}\right) \\
& =\sum_{k=1}^{m} \frac{2(m)_{k-1}}{(2 \pi i n)^{k}}\left(C h_{m-k+1}^{(r)}-C h_{m-k+1}^{(r-1)}\right) .
\end{aligned}
$$

Here, we used the fact that

$$
C h_{1}^{(r)}-C h_{1}^{(r-1)}=r C h_{1}-(r-1) C h_{1}=C h_{1}=-\frac{1}{2} .
$$


Indeed,

$$
\begin{aligned}
\sum_{n=0}^{\infty} C h_{n}^{(r)} \frac{t^{n}}{n !} & =\left(\frac{2}{2+t}\right) \times \cdots \times\left(\frac{2}{2+t}\right) \\
& =\sum_{n=0}^{\infty}\left(\sum_{l_{1}+\cdots+l_{r}=n}\left(\begin{array}{c}
n \\
l_{1}, l_{2}, \ldots, l_{r}
\end{array}\right) C h_{l_{1}} C h_{l_{2}} \cdots C h_{l_{r}}\right) \frac{t^{n}}{n !} .
\end{aligned}
$$

Accordingly, it follows that

$$
\begin{aligned}
C h_{1}^{(r)} & =\sum_{l_{1}+\cdots+l_{r}=1}\left(\begin{array}{c}
1 \\
l_{1}, l_{2}, \ldots, l_{r}
\end{array}\right) C h_{l_{1}} C h_{l_{2}} \cdots C h_{l_{r}} \\
& =C h_{1}+C h_{1}+\cdots+C h_{1}=r C h_{1} .
\end{aligned}
$$

Case 2 Let $n=0$. Then we have

$$
\begin{aligned}
C_{0}^{(r, m)} & =\int_{0}^{1} C h_{m}^{(r)}(x) d x \\
& =\frac{1}{m+1}\left[C h_{m+1}^{(r)}(1)-C h_{m+1}^{(r)}\right]_{0}^{1} \\
& =\frac{2}{m+1}\left(C h_{m+1}^{(r-1)}-C h_{m+1}^{(r)}\right) .
\end{aligned}
$$

Assume first that $C h_{m}^{(r)}(1)=C h_{m}^{(r)}$. Then we have $C h_{m}^{(r)}(1)=C h_{m}^{(r)}$ for $m \geq 2 . C h_{m}^{(r)}(\langle x\rangle)$ is piecewise $C^{\infty}$ and continuous. Hence the Fourier series of $C h_{m}^{(r)}(\langle x\rangle)$ converges uniformly to $C h_{m}^{(r)}(\langle x\rangle)$, and

$$
\begin{aligned}
C h_{m}^{(r)}(\langle x\rangle)= & \frac{2}{m+1}\left(C h_{m+1}^{(r-1)}-C h_{m+1}^{(r)}\right) \\
& +\sum_{\substack{n=-\infty \\
n \neq 0}}\left[\sum_{k=1}^{m} \frac{2(m)_{k-1}}{(2 \pi i n)^{k}}\left(C h_{m-k+1}^{(r)}-C h_{m-k+1}^{(r-1)}\right)\right] e^{2 \pi i n x} .
\end{aligned}
$$

Consequently, it follows that

$$
\begin{aligned}
C h_{m}^{(r)}(\langle x\rangle)= & \frac{2}{m+1}\left(C h_{m+1}^{(r-1)}-C h_{m+1}^{(r)}\right) \\
& +\sum_{k=1}^{m} \frac{2(m)_{k-1}}{k !}\left(C h_{m-k+1}^{(r-1)}-C h_{m-k+1}^{(r)}\right) \sum_{\substack{n=-\infty \\
n \neq 0}}(-k !) \frac{e^{2 \pi i n x}}{(2 \pi i n)^{k}} \\
= & \frac{2}{m+1}\left(C h_{m+1}^{(r-1)}-C h_{m+1}^{(r)}\right) \\
& +\sum_{k=2}^{m} \frac{2(m)_{k-1}}{k !}\left(C h_{m-k+1}^{(r-1)}-C h_{m-k+1}^{(r)}\right) B_{k}(\langle x\rangle) \\
& +2\left(C h_{m}^{(r-1)}-C h_{m}^{(r)}\right) \times \begin{cases}B_{1}(\langle x\rangle) & \text { for } x \notin \mathbb{Z}, \\
0 & \text { for } x \in \mathbb{Z} .\end{cases}
\end{aligned}
$$

Thus the proof of Theorem 3 is complete. 
Finally, assume that $C h_{m}^{(r)} \neq C h_{m}^{(r-1)}$. Then we have $C h_{m}^{(r)}(1) \neq C h_{m}^{(r)}$ and hence $C h_{m}^{(r)}(\langle x\rangle)$ is piecewise $C^{\infty}$ and discontinuous with jump discontinuities at integers. Thus the Fourier series of $C h_{m}^{(r)}(\langle x\rangle)$ converges pointwise to $C h_{m}^{(r)}(\langle x\rangle)$ for $x \notin \mathbb{Z}$, and converges to $\frac{1}{2}\left(C h_{m}^{(r)}+\right.$ $\left.C h_{m}^{(r)}(1)\right)=C h_{m}^{(r-1)}$ for $x \in \mathbb{Z}$. From the above considerations, the proof of Theorem 4 is complete.

\section{Conclusions}

In this paper, the author considered the Fourier series expansion of the higher-order Daehee functions $D_{n}^{(r)}(\langle x\rangle)$ and the higher-order Changhee functions $C h_{n}^{(r)}(\langle x\rangle)$ which are obtained by extending by periodicity of period 1 the higher-order Daehee polynomials $D_{n}^{(r)}(x)$ and the higher-order Changhee polynomials $C h_{n}^{(r)}(x)$ on $[0,1)$, respectively. The Fourier series are explicitly determined. Depending on whether $D_{n}^{(r)}(\langle x\rangle)$ and $C h_{n}^{(r)}(\langle x\rangle)$ are zero or not, the Fourier series of these functions converge uniformly or converge pointwise. In addition, the Fourier series of the higher-order Daehee functions $D_{n}^{(r)}(\langle x\rangle)$ and the higherorder Changhee functions $C h_{n}^{(r)}(\langle x\rangle)$ are expressed in terms of the Bernoulli functions $B_{k}(\langle x\rangle)$. Thus we established the relations between these functions and Bernoulli functions.

\section{Acknowledgements}

The author wishes to express his sincere gratitude to the referees for their valuable suggestions and comments. This work is supported by China Postdoctoral Science Foundation (2016M591379).

\section{Competing interests}

The author declares that he has no competing interests.

\section{Author's contributions}

The author carried out all work of this article and the main theorem. The author read and approved the final manuscript.

\section{Publisher's Note}

Springer Nature remains neutral with regard to jurisdictional claims in published maps and institutional affiliations.

Received: 14 April 2017 Accepted: 14 June 2017 Published online: 24 June 2017

References

1. Abramowitz, M, Stegun, IA: Handbook of Mathematical Functions. Dover, New York (1970)

2. Berndt, BC: Periodic Bernoulli numbers, summation formulas and applications. In: Theory and Application of Special Functions (Proc. Advanced Sem., Math. Res. Center, Univ. Wisconsin, Madison, Wis., 1975). Publication of the Mathematics Research Center, the University of Wisconsin, vol. 35, pp. 143-189. Academic Press, New York (1975)

3. Carlitz, L: A note on Bernoulli numbers and polynomials. Elem. Math. 29, 90-92 (1974)

4. El-Desouky, BS, Mustafa, A: New results on higher-order Daehee and Bernoulli numbers and polynomials. Adv. Differ. Equ. 2016, Article ID 32 (2016)

5. Gould, HW: Explicit formulas for Bernoulli numbers. Am. Math. Mon. 79, 44-51 (1972)

6. Herget, W: Minimum periods modulo $n$ for Bernoulli numbers. Fibonacci Q. 16(6), 544-548 (1978)

7. Jang, G-W, Kim, T, Kim, DS, Mansour, T: Fourier series of functions related to Bernoulli polynomials. Adv. Stud. Contemp. Math. 27(1), 49-62 (2017)

8. Kim, T: Euler numbers and polynomials associated with zeta functions. Abstr. Appl. Anal. 2008, Article ID 581582 (2008)

9. Kim, DS, Kim, T: Daehee numbers and polynomials. Appl. Math. Sci. (Ruse) 7(117-120), 5969-5976 (2013)

10. Kim, DS, Kim, T: Identities arising from higher-order Daehee polynomial bases. Open Math. 13, 196-208 (2015)

11. Kim, T, Kim, DS, Dolgy, D, Park, J-W: Fourier series of sums of products of poly-Bernoulli functions and their applications. J. Nonlinear Sci. Appl. 10(4), 2384-2401 (2017)

12. Kim, T, Kim, DS, Dolgy, D, Park, J-W: Fourier series of sums of products of ordered Bell and poly-Bernoulli functions. J. Inequal. Appl. 2017, Article ID 84 (2017)

13. Kim, T, Kim, DS, Jang, L-C, Jang, G-W: Fourier series of sums of products of Bernoulli functions and their applications. J. Nonlinear Sci. Appl. 10(5), 2798-2815 (2017)

14. Kim, T, Kim, DS, Komatsu, T, Lee, S-H: Higher-order Daehee of the second kind and poly-Cauchy of the second kind mixed-type polynomials. J. Nonlinear Convex Anal. 16(10), 1993-2015 (2015)

15. Kim, T, Kim, DS, Rim, S-H, Dolgy, DV: Fourier series of higher-order Bernoulli functions and their applications. J. Inequal. Appl. 2017, Article ID 8 (2017)

16. Lim, D, Qi, F: On the Appell type $\lambda$-Changhee polynomials. J. Nonlinear Sci. Appl. 9(4), 1872-1876 (2016) 
17. Seo, J-J, Rim, S-H, Kim, T, Lee, SH: Sums products of generalized Daehee numbers. Proc. Jangjeon Math. Soc. 17(1), 1-9 (2014)

18. Shiratani, K: Kummer's congruence for generalized Bernoulli numbers and its applications. Mem. Fac. Sci., Kyushu Univ., Ser. A, Math. 26, 119-138 (1972)

19. Simsek, Y: Apostol type Daehee numbers and polynomials. Adv. Stud. Contemp. Math. (Kyungshang) 26(3), 555-566 (2016)

20. Yilmaz Yasar, B, Özarslan, MA: Frobenius-Euler and Frobenius-Genocchi polynomials and their differential equations. New Trends Math. Sci. 3(2), 172-180 (2015)

21. Vasak, JT: Periodic Bernoulli numbers and polynomials. PhD thesis, University of Illinois at Urbana-Champaign (1979)

22. Washington, LC: Introduction to Cyclotomic Fields, 2nd edn. Graduate Text in Mathematics, vol. 83. Springer, New York (1997)

23. Wang, NL, Li, H: Some identities on the higher-order Daehee and Changhee numbers. Pure Appl. Math. J. 5, 33-37 (2015)

24. Cohen, H: Number Theory Volume II: Analytic and Modern Tools. Graduate Text in Mathematics, vol. 240. Springer, New York (2007)

Submit your manuscript to a SpringerOpen ${ }^{\circ}$ journal and benefit from:

- Convenient online submission

Rigorous peer review

- Open access: articles freely available online

- High visibility within the field

- Retaining the copyright to your article

Submit your next manuscript at $\gg$ springeropen.com 